\title{
An Environmental-Economic Dispatch Method for Smart Microgrids Using VSS_QGA
}

\author{
Wenxia Liu, ${ }^{1}$ Yuying Zhang, ${ }^{1}$ Bo Zeng, ${ }^{1}$ Shuya Niu, ${ }^{1}$ Jianhua Zhang, ${ }^{1}$ and Yong Xiao ${ }^{2}$ \\ ${ }^{1}$ Transmission and Distribution System Research Institute, North China Electric Power University, \\ Number 2 Beinong Road, Changping District, Beijing 102206, China \\ ${ }^{2}$ Guizhou Electric Power Test Research Institute, China Southern Power Grid, Number 32 Jiefang Road, \\ Nanming District, Guiyang 550000, China \\ Correspondence should be addressed to Yuying Zhang; zhyuying@126.com
}

Received 24 January 2014; Revised 16 March 2014; Accepted 23 March 2014; Published 11 May 2014

Academic Editor: Hongjie Jia

Copyright (C) 2014 Wenxia Liu et al. This is an open access article distributed under the Creative Commons Attribution License, which permits unrestricted use, distribution, and reproduction in any medium, provided the original work is properly cited.

\begin{abstract}
The increasing penetration of distributed generation resources demands better economic performance of microgrids under the smart-grid era. In this paper, a comprehensive environmental-economic dispatch method for smart microgrids is proposed, with the objective for minimizing the summation of generation and emission costs in the system. As the proposed model belongs to a large-scale nonlinear and nonconvex programming problem, a hybrid heuristic algorithm, named variable step-size chaotic fuzzy quantum genetic algorithm (VSS_QGA), is developed. The algorithm utilizes complementarity among multiple techniques including the variable step size optimization, the rotation mutational angle fuzzy control, and the quantum genetic algorithm and combines them so as to solve problems with superior accuracy and efficiency. The effectiveness of the proposed model is demonstrated through a case study on an actual microgrid system and the advantages in the performance of VSS_QGA is also verified through the comparison with genetic algorithm (GA), the evolutionary programming approach (EP), the quantum genetic algorithm (QGA), and the chaotic quantum genetic algorithm (CQGA).
\end{abstract}

\section{Introduction}

A microgrid (MG) is defined as an electrical system that includes multiple loads and distributed energy resources, which can be operated in grid-connected or islanding mode. Due to its superior economic efficiency and operational flexibility [1], MG has been widely regarded as a novel energy supply form under the smart-grid paradigm. If applied properly, MG is beneficial to network investment deferral, pollution reduction, and improvement of energy utilization efficiency and supply reliability $[2,3]$.

During the 13th five-year plan period, distributed generation (DG) will undergo development in China. With increasing DG penetration into MG, the issue of "how to obtain the optimal dispatch balancing the controversy between economic and environmental benefits while meeting the load demand and network security" has become an urgent problem for MG operators.
Due to the differences in the operation characteristics in bulk power systems and MGs, there are significant distinctions in their objectives and dispatching strategies. First, the dispatch of traditional grid usually focuses on economy for optimization, through dispatching different types of thermal power unit to achieve real-time balance of the network power. Contrast to the main grid, there are lots of uncontrollable renewable distributed generations (RDG) in MG. Only the conventional units and power purchasing from the main grid are control variables. Second, when multiple unfirm generators are connected, the network capacity is shared based on a particular principle of access (PoA) in order to balance economic and environmental benefits and maintain the safe and stable operation of the system. Third, in traditional power system, the single-period based optimal power flow (OPF) is widely used as a suitable tool for power dispatch problem, due to the lack of RDG units [4]. The MG integrates a large number of RDGs and requires dynamic optimal power flow 
(DOPF) to treat intermittent and temporal correlation of $\mathrm{DG}$. Due to the difference between the dispatching of traditional grid and $M G$, it is of great significance to investigate the suitable dispatch method for smart MG.

Recently, a number of optimization models and algorithms for economic dispatch (ED) of MG with different types of DG units have been developed [5-7]. In [8], a penalty function-hybrid direct search method (PF-HDSM) was developed to allocate the active power output of multiarea wind-thermal generating units to minimize the total operating cost while fulfilling the equality and inequality constraints. For the same purpose, a simple and efficient harmony search (HS) method with a new pitch adjustment rule (NPAHS) was proposed for dynamic economic dispatch (DED) of electrical power systems in [6]. And a hybrid solution methodology involving modified shuffled frog leaping algorithm (MSFLA) with genetic algorithm (GA) was carried out by [7] in which the valve-point effect was considered. With the increasing public awareness of environmental protection, it has become essential to optimize not only the fuel cost but also the emission levels of harmful pollutants. In this regard, ED problems will become economic emission dispatch (EED) problems when in addition to it there are environmental concerns caused by emissions from fossil-fuel electric power plants [9]. The EED problem is a nonlinear biobjective optimization subject designed to deal with minimization of two conflicting objectives, namely, fuel cost and air pollution from thermal power plants simultaneously [8]. The consideration of environmental emissions in MG operations could render optimization being a nonlinear and nonconvex problem with plenty of local optima. It is difficult to reach the global optimal solution with conventional numerical analysis method, such as Lambda iteration and gradient methods [3]. Recently, some heuristic algorithms have been applied to EED problems in MG, such as virus optimization algorithm (VOA) [10], hybrid particle swarm optimization and gravitational search algorithm (HPSO-GSA) [11], incremental artificial bee colony algorithm with local search (IABC-LS) [4], or $\varepsilon$-multi-objective GA variable ( $\varepsilon$ v-MOGA) [12]. However, the above algorithms lack adaptability for large scale systems, and the calculation speed and convergence accuracy still need to be improved. Therefore, developing more sophisticated algorithms for dispatch optimization of MG is necessary.

GA is a classical algorithm that is used to solve the combinational optimization problem or nonlinear optimization problem with nondifferentiable objective function or complicated constraint condition. Due to its parallel computing character and the ability of adaptive search, GA has been proposed for the solution of EED problems [13]. However, GA also shows more iterative times and slow convergence speed and easily falls into local optimum. In 1996, Ajit Narayanan and Mark Moore proposed quantum genetic algorithm (QGA) which introduced the quantum theory into genetic algorithm. Due to its stronger search capability and more efficient and simple characteristics, QGA has been proven to be a favorable technique to tackle ED/EED problems of power systems [14]. In spite of its improvements, QGA has its shortages like premature convergence and, similarly, being caught in local optimum. To improve this problem, an advanced version with superior accuracy and efficiency is developed.

An EED model for smart microgrids (SMG) is proposed in this paper, which aimed to minimize the generation and emission cost. As the proposed model belongs to a largescale nonlinear and nonconvex programming problem, a hybrid heuristic algorithm, named variable step-size chaotic fuzzy QGA (VSS_QGA), is developed. The algorithm combines complementarity among several techniques such as the variable step size optimization, the rotation mutation angle fuzzy control, and the quantum genetic algorithm to solve problems with superior accuracy and efficiency. For that very reason, it can be immune to local optima and has an improved searching efficiency compared to cases where the techniques are each used individually. The effectiveness of the proposed model is demonstrated through a case study on an actual MG system and the advantages in the performance of VSS_QGA are also confirmed through comparison with genetic algorithm (GA), evolutionary programming approach (EP), quantum genetic algorithm (QGA), and chaotic quantum genetic algorithm (CQGA).

\section{Model Formulation for EED of SMG}

In general, it is assumed that the SMG under study is composed of three different types of DG, namely, wind turbines (WT), solar photovoltaic (PV), and diesel internal combustion engine (DICE). As the power output of WT and $\mathrm{PV}$ units is mainly dependent on the primary renewable energy supply, the goal of EED is to allocate the active power of controllable DG units (i.e., DICE) that was installed and to achieve minimizing the generation and emission cost of the entire system while meeting customer load demand and network Figure 4.

2.1. Objective Function. The objective function of the proposed EED model for SMG is given as follows:

$$
\text { Min } C_{\text {sum }}=(1-v) C_{\text {gen }}+\nu C_{\text {emission }}
$$

subject to

$$
\begin{gathered}
C_{\text {gen }}=\sum_{t=1}^{T}\left[\sum_{i=1}^{n} C_{n \mathrm{dg}}\left(p_{i, t}^{n \mathrm{dg}}\right)+\sum_{j=1}^{m} \theta_{j} p_{j, t}^{d \mathrm{reg}}+\theta_{t} p_{t}^{\mathrm{gsp}}\right], \\
C_{\text {emission }}=\sum_{t=1}^{T}\left[\sum_{a=1}^{A} C_{\mathrm{env}, a}\left(p_{i, t}^{n \mathrm{dg}}\right)+\sum_{a=1}^{A} \Lambda_{\mathrm{env}, a}\left(p_{t}^{\mathrm{gsp}}\right)\right] .
\end{gathered}
$$

In above equation, the objective function $C_{\text {sum }}$ is composed of both expected operating cost $C_{\text {gen }}$ and emission cost $C_{\text {emission }} v$ is a coefficient representing the importance of environmental value and $1-v$ and $v$ are penalty factors of $C_{\text {gen }}$ and $C_{\text {emission }}$, respectively. Different values of $\nu$ mean different importance between environment and economy, which then lead to different optimization results. Operating personnel can recognize flexible tradeoffs by adjusting these coefficients. $T$ is the number of time periods in a scheduling interval. With 
regard to the regulation of day-ahead generation scheduling, $T$ can be considered to be 24 hours. $n$ is the number of controllable DICE units in the MG; $m$ is the number of distributed renewable energy generation (DREG) in MG. $p_{i, t}^{n \mathrm{dg}}$ and $p_{j, t}^{d \mathrm{reg}}$ are the scheduled active power outputs from unit $i$ of DICE and unit $j$ of DERG in period $t$, respectively; $p_{t}^{\mathrm{gsp}}$ is the active power injected from main grid in period $t$; $\theta_{j}$ and $\theta_{t}$ are spot prices of purchasing power from DREG and main grid during period $t$, respectively $(¥ / \mathrm{kWh}) ; C_{n \mathrm{dg}, i}(\bullet)$ represents the generation cost function of DICE unit $i$, which is calculated as follows:

$$
C_{n \mathrm{dg}}\left(p_{i, t}^{n \mathrm{dg}}\right)=\left[\alpha_{i}\left(p_{i, t}^{n \mathrm{dg}}\right)^{2}+\beta_{i} p_{i, t}^{n \mathrm{dg}}+\delta_{i}\right]+\mu_{i} p_{i, t}^{n \mathrm{dg}} .
$$

In above equation, $\alpha_{i}\left(p_{i, t}^{n \mathrm{dg}}\right)^{2}+\beta_{i} p_{i, t}^{n \mathrm{dg}}+\delta_{i}$ represents the fuel cost for per kilowatt-hour ( $¥ / \mathrm{kWh}$ ) of electricity produced by DICE unit $i$, where $\alpha_{i}, \beta_{i}$, and $\delta_{i}$ are the coefficients of fuel cost function; $\mu_{i}$ is the rate for operation and maintenance of DICE unit $i(¥ / \mathrm{kWh})$.

In addition, " $A$ " in (3) represents the set of emission pollutants due to generation; $C_{\mathrm{env}, a}(\bullet)$ and $\Lambda_{\mathrm{env}, a}(\bullet)$ are the emission cost function of DICE and main grid with respect to pollution $a$, respectively, $(\mathrm{kg} / \mathrm{kWh})$, which is calculated as follows:

$$
\begin{gathered}
C_{\mathrm{env}, a}\left(p_{i, t}\right)=\xi_{d e, a, i} \times H_{d e, a} \times p_{i, t}^{n \mathrm{dg}}, \\
\Lambda_{\mathrm{env}, a}\left(p_{\mathrm{gsp}, t}\right)=\xi_{\mathrm{gsp}, a} \times H_{d e, a} \times p_{t}^{\mathrm{gsp}},
\end{gathered}
$$

where $\xi_{d e, a, i}$ and $\xi_{\mathrm{gsp}, a}$ are emission intensity from unit $i$ of DICE and main grid $(\mathrm{t} / \mathrm{kWh})$, respectively, representing the amount of emission induced for producing per kilowatt-hour of pollutant $a . H_{d e, a}$ is the charge rate for emission of pollutant $a(¥ / t)$.

In the objective function (1)-(4), despite of the introduction of the coefficient $v$, the tradeoff between economic and environmental motivations can be achieved, which represents the coordinated MG operation towards the lowcarbon economy.

2.2. Constraints. The optimization formula of EED in (1) is subject to the following set of equality and inequality constraints, as shown in (7)-(13).

\subsubsection{Power Balance Constraint. Consider}

$$
\sum_{i=1}^{n} p_{i, t}^{n \mathrm{dg}}+p_{t}^{\mathrm{gsp}}=p_{t}^{l}+p_{t}^{\mathrm{loss}}-\sum_{j=1}^{n_{w}} p_{j, t}^{\mathrm{wind}}-\sum_{k=1}^{n_{s}} p_{k, t}^{\mathrm{pv}},
$$

where $p_{t}^{l}$ and $p_{t}^{\text {loss }}$ are predicted value of load demand and network losses in the period $t ; p_{j, t}^{\text {wind }}$ and $p_{k, t}^{\mathrm{pv}}$ represent the expected power output of WG and PV in period $t$, respectively; $n_{w}$ and $n_{s}$ are the number of WG and PV units installed in the SMG.

In the SMG, RDG units are integrated into the network based on the "fit-and-forget" strategy, which is lack of controllability and visibility. Optimizing the operation of these resources requires consideration of intertemporal linkages as well as network power flow solutions. But this only optimizes at a single point of time. DOPF is an extension of OPF to cover multiple time periods. DOPF breaks the time-horizon into $t_{n}$ time-steps and extends all OPF variables and some parameters into time series. The network must obey the typical OPF constraints such as power flow equations during each time-step independently.

2.2.2. Power Output Limits of Controllable DG Units. To guarantee safe and stable operation of a MG, the power outputs of DG units in the MG must fall into their lower and upper limits in the operation capacity, as shown in (6)-(7):

$$
\begin{aligned}
& p_{i, \text { min }}^{n \mathrm{dg}} \leq p_{i}^{n \mathrm{dg}} \leq p_{i, \text { max }}^{n \mathrm{dg}}, \\
& q_{i, \text { min }}^{n \mathrm{dg}} \leq q_{i}^{n \mathrm{dg}} \leq q_{i, \text { max }}^{n \mathrm{dg}},
\end{aligned}
$$

where $p_{i, \text { max }}^{n \mathrm{dg}} / p_{i, \text { min }}^{n \mathrm{dg}}$ are the upper and lower boundaries for the active power output of controllable DG $i$, respectively; likewise, $q_{i, \max }^{n \mathrm{dg}} / q_{i, \text { min }}^{n \mathrm{dg}}$ denote the upper and lower limits for its reactive power output.

2.2.3. Power Output Limits of RDG Units. As renewable energy sources generally do not provide reactive power to the system, there is only one constraint concerning the active power output for each RDG unit, expressed as follows:

$$
p_{i, \text { min }}^{r \mathrm{dg}} \leq p_{i}^{r \mathrm{dg}} \leq p_{i, \text { max }}^{r \mathrm{dg}}
$$

where $p_{i, \text { max }}^{r \mathrm{dg}} / p_{i, \text { min }}^{r \mathrm{dg}}$ are the upper and lower limits for the active power output of RDG unit $i$, respectively.

2.2.4. Ramp Rate Limits of Controllable DG. In actual practice, the system operator typically makes energy acquisition plan on an hourly basis. As the load on system changes in subsequent planning intervals, the power output of controllable DG units is changed to match the load. However, this change does not take place instantaneously whenever load changes. Therefore, it is necessary to incorporate the following ramprate limits in the EED model:

$$
R_{i}^{d} \Delta t \leq p_{i, t}^{n \mathrm{dg}}-p_{i, t-1}^{n \mathrm{dg}} \leq R_{i}^{u} \Delta t,
$$

where $\Delta t$ represents the duration of time periods; $R_{i}^{u}, R_{i}^{d}$ are the up and down ramp rate limits of the controllable DG $i$, respectively.

2.2.5. Power Interaction Limits of Smart MG with Main Grid. DREG and main grid jointly participate in the optimal operation of power system. When power shortage happens in SMG, it can absorb power from main grid in real time but can be subjected to the maximum capacity constraints of circuits and the system

$$
p_{i, \min }^{\mathrm{gsp}} \leq p_{i}^{\mathrm{gsp}} \leq p_{i, \max }^{\mathrm{gsp}}
$$

where $p_{i, \text { max }}^{\mathrm{gsp}} / p_{i, \min }^{\mathrm{gsp}}$ are the upper and lower boundaries for the power interacted between SMG and main grid.

Furthermore, the network constraints should be also taken into consideration. 
2.2.6. Thermal Limit of Feeders. The magnitude of current passing through the feeders of a system should be within their thermal limit during operation, which is expressed as

$$
0 \leq\left|I_{m n}\right| \leq I_{m n, \max }
$$

where $I_{m n \text {, max }}$ is the permitted maximum current limit in the feeder $m n$ in the time period $t$.

2.2.7. Node Voltage Constraint. To ensure the quality of electricity supply, the voltage variation for each node in the system must satisfy the following constraint, as is presented below:

$$
V_{m, \min } \leq \Delta V_{m} \leq V_{m, \max }
$$

where $V_{m, \min }$ and $V_{m, \max }$ stand for the minimum and maximum limits with respect to the voltage variation.

\section{Variable Step-Size Chaotic Fuzzy Quantum Genetic Algorithm}

Chaotic quantum genetic algorithm (CQGA) is an improved version of the well-known quantum evolutionary algorithm (QEA) [15], which incorporates the global searching ability of GA, the local search ability of quantum probability technique, and the adaptability and transverse mobile ability of chaotic algorithm. Hence, CQGA is widely acknowledged as an effective tool to solve EED problem.

To construct VSS_QGA, we take into consideration the effect of initial value selection, the search step size on the convergence speed, and the calculation precision. It combines the variable step size optimal method, the rotation mutational angle fuzzy control method, and the quantum genetic algorithm to obtain the global optimal solution with a fast search speed and higher calculation accuracy. The steps for VSS_QGA in EED are described as follows.

Step 1. Initialize populations with the variable step size chaos algorithm.

(1) Chaotic System. Chaos is a universal, nonlinear phenomenon in nature. Its behavior is complex and similar to being random. However, due to the existence of the faint positive characteristic, a chaotic system optimizes general chaos variables by using logistic mapping:

$$
x_{j+1}=\lambda x_{j}\left(1-x_{j}\right),
$$

where $\lambda$ is a chaotic attractor; $j$ is the number of iterations. When $\mu=4$, the system gets into chaotic state, producing the chaos variables $x_{j}(j=1,2,3, \ldots)$, between $(0,1)$.

After $j$ iterations, $x_{i j}(i=1,2,3, \ldots, k)$ are obtained in which $k$ is the variable dimension. The second carrier wave can be obtained by using the following formula:

$$
x_{i}\left(m^{\prime}\right)=x_{i}^{*}+\eta_{i}\left(x_{i, j+m}-0.5\right),
$$

where $x_{i}\left(m^{\prime}\right)$ is the chaos variable in the smaller ergodic interval of the feasible region relative to the optimization objective function $\mathrm{TC}(\cdot) . \eta_{i}$ is the adjustment coefficient. $x_{i}^{*}$ is an optimal solution in the initial stage of searching.

For the initialization of the chaos system, if $m=1, m^{\prime}=1$, $x_{i}(m)=x_{i}(0)$, optimal solution $x_{i}^{*}=x_{i}(0)$, and the minimum of optimization objective function $\mathrm{TC}_{i}^{*}=\mathrm{TC}(0)$, the upper and lower ranges of optimization variables are $A_{i}\left(m^{\prime}\right)=A_{i}$ and $B_{i}\left(m^{\prime}\right)=B_{i}$, respectively. The chaotic variable $x_{i}(m)$ is mapped into the optimization variables space. The general method of chaos is used to search by making $m=m+1$, $x_{i}(m)=4 x_{i}(m)\left[1-x_{i}(m)\right]$, until the local optimal value does not change. At this stage, the step size in search is changed to the following equations:

$$
\begin{aligned}
& A_{i}\left(m^{\prime}+1\right)=k x_{i}^{*}-\gamma\left(B_{i}\left(m^{\prime}\right)-A_{i}\left(m^{\prime}\right)\right), \\
& B_{i}\left(m^{\prime}+1\right)=k x_{i}^{*}+\gamma\left(B_{i}\left(m^{\prime}\right)-A_{i}\left(m^{\prime}\right)\right),
\end{aligned}
$$

where $\gamma \in(0,0.5)$ is the adjustment coefficient; $k x_{i}^{*}$ is the current optimal solution.

The optimization variables are restored to be

$$
x_{i}^{*}=k x_{i}^{*}-A_{i}\left(m^{\prime}+1\right) B_{i}\left(m^{\prime}+1\right)-A_{i}\left(m^{\prime}+1\right) .
$$

Through the new chaotic variable $x_{i}^{\prime}(m)=(1-\delta) x_{i}^{*}+$ $\delta x_{i}(m)$ ( $\delta$ is a smaller number), we repeat the above steps of chaotic searching and make $m^{\prime}=m^{\prime}+1$ until the minimum of optimization objective function does not change. After several iterations, the optimal variable $k x_{i}^{*}$ and the corresponding optimal objective function will be obtained by this algorithm.

(2) Quantum Bit. In CQGA, the smallest information unit is quantum bit (qubit). A qubit state can be $|0\rangle$ or $|1\rangle$, which can be expressed as

$$
|\Psi\rangle=\alpha|0\rangle+\beta|1\rangle
$$

where $\alpha, \beta$ are tow complex of probability amplitude of the corresponding state, where $|a|^{2}+|b|^{2}=1\left(|a|^{2}\right.$ and $|b|^{2}$ represent the occurrence probabilities of qubit in the states of $|0\rangle$ and $|1\rangle$, respectively).

(3) Quantum Chromosome. The commonly used coding algorithms in evolutionary algorithm (EA) are binary, decimal, and symbolic coding. In the CQGA, a new coding method based on quantum bit is determined by a pair of complex number. The system with $e$ quantum bits can be expressed as follows [16]:

$$
\left[\begin{array}{llll}
a_{1} & a_{2} & \cdots & a_{e} \\
b_{1} & b_{2} & \cdots & b_{e}
\end{array}\right],
$$

where $\left|a_{h}\right|^{2}+\left|b_{h}\right|^{2}=1(h=1,2, \ldots, e)$. This expression can be used to represent any linear superposition state between $|0\rangle$ and $|1\rangle$.

(4) Chaotic Quantum Populations. Using the following $n$ units of the logistic mapping produces $n$ units of chaotic variables as

$$
x_{j+1, l}=\mu_{l} \times x_{j, l} \times\left(1-x_{j, l}\right)
$$


where $\mu_{l}=4 ; l$ is the sequence number of chaotic variables. If $j=0$, a set of different initial values to a given $n$ units of chaotic variables can be obtained. Chaotic variables of $n$ units can also be obtained by using $(20)(l=1,2, \ldots, n)$. Using the first qubit of the number $n$ chaotic variable initialization populations, by making $j=1,2, \ldots, n-1$ based on Step 1 , we can obtain another $n-1$ solutions. These $n$ solutions will consist of the initial populations.

Step 2. The encoding and individual measurements to the power output of generator in populations CQGA and EA are probability algorithms. In the population, $G_{t}=$ $\left\{Q_{t, 1}, Q_{t, 2}, \ldots, Q_{t, s}, \ldots, Q_{t, M}\right\}(s=1,2, \ldots, M)$, where $t$ is the generation of evolutionary and $s$ is the size of population; $Q_{t, s}$ is the individual of number $t$ generation. In $Q_{t}=\left\{q_{t, 1}, q_{t, 2}, \ldots, q_{t, i}, \ldots, q_{t, n}\right\}, q_{t, i}(i=1,2, \ldots, n)$ is the binary code of number $i$ unit power generation in number $t$ generation. The chromosome is defined as (21)

$$
q_{t, i}=\left[\begin{array}{llll}
a_{t, 1} & a_{t, 2} & \ldots & a_{t, e} \\
b_{t, 1} & b_{t, 2} & \ldots & b_{t, e}
\end{array}\right], \quad i=1,2, \ldots, n,
$$

where $e$ represents the length of the quantum chromosome.

In the initial group $G_{t}$, if $a_{t, h}, b_{t, h}(h=1,2, \ldots, e)$ and all the $q_{1, i}$ in $Q_{1}$ have been initialized, that is, all the possible linear superposition states could emerge with the same probability.

In the process of generating solution sets from $G_{t}$, a general solution set, $S_{t}=\left\{q_{t, 1}, q_{t, 2}, \ldots, q_{t, s}, \ldots, q_{t, M}\right\}$, can be obtained $\left(q_{t}=\left\{q_{t, 1}, q_{t, 2}, \ldots, q_{t, i}, \ldots, q_{t, n}\right\}\right)$; for each $q_{t, i}(i=$ $1,2, \ldots, n)$, whose length is $e$, there is a binary character string $\left(x_{1}, x_{2}, \ldots, x_{h}, \ldots, x_{e}\right)$. Through the range of quantum bits $\left|a_{t, h}\right|^{2}$ and $\left|b_{t, h}\right|^{2} \quad(h=1,2, \ldots, e)$, it can be found that in the binary coding a number $w$ between $[0,1]$ will be randomly generated. In addition, if $\left|a_{t, h}\right|^{2}>1,1$ will be taken; otherwise, the 0 will be taken.

Step 3. Evaluate individual targets in $S_{t}$ separately.

Individual targets in $S_{t}$ are evaluated through a fitness function to ensure that the total generation cost reaches the optimal target. If a satisfactory solution is obtained, the algorithm terminates. Otherwise, it moves on to Step 4.

Step 4. Update $S_{t}$ by using an appropriate quantum rotating gate $V_{t}$.

The QGA applies logic gates into the quantum probability amplitude to maintain diversity of the population. Therefore, updating method using a quantum gate is the key to the QGA. In principle, this updating method applies to find solutions for combinatorial optimization problems through known optimal solution. However, for practical optimization problems, in particular those multivariable continuous function optimization problems, their optimal solutions are not obtained beforehand. Therefore, quantum rotation gates of quantum logic gates are adopted for the QGA:

$$
U=\left[\begin{array}{cc}
\cos \omega & -\sin \omega \\
\sin \omega & \cos \omega
\end{array}\right]
$$

where $\omega$ is the rotation variation angle of the quantum gate.
Rotation of the control quantum gates on the rotating angle is used to update the quantum bits in this paper, as described in the following:

$$
\left[\begin{array}{l}
a_{i} \\
b_{i}
\end{array}\right]=\left[\begin{array}{cc}
\cos \Delta \omega & -\sin \Delta \omega \\
\sin \Delta \omega & \cos \Delta \omega
\end{array}\right]\left[\begin{array}{l}
a_{i} \\
b_{i}
\end{array}\right],
$$

where $\Delta \omega$ is the rotation variation angle, with convergence improved by controlling the amplitude of $\Delta \omega$. If the amplitude of $\Delta \omega$ is too high, the problem of premature convergence occurs, and $\Delta \omega$ will be constant. To make full use of the trend of fitness function $F(\cdot)$ and adjust the scale of MG automatically, $\Delta \omega$ needs to change accordingly; therefore, QGA must consider the function value and the rate of change near the individual chromosome in order to adopt the changes of the fitness function into the rotation angle step function, as described in the following:

$$
\begin{aligned}
\omega= & \omega_{0} \times \frac{\rho \cdot\left\|F_{\max }-F_{\min }\right\|}{\left\|F(X)-F_{\min }\right\|} \\
& +\frac{(1-\rho) \cdot\left\|\Delta F_{\max }-\Delta F_{\min }\right\|}{\left\|\Delta F(X)-\Delta F_{\min }\right\|},
\end{aligned}
$$

where $\omega_{0}$ is the initial value of iteration. $\rho \in[0,1]$ is the control factor. $F_{\max }$ and $F_{\min }$ are the minimum and maximum values of fitness function, respectively; $\Delta F(X)$ is the gradient at point $X$ of the fitness function $F(\cdot) . \Delta F_{\max }$ and $\Delta F_{\min }$ are, respectively, determined by the following equation [17]:

$$
\begin{aligned}
\Delta F_{\max }= & \left\{\max \left[\frac{\partial F\left(X_{1}\right)}{\partial X_{1}^{1}}, \frac{\partial F\left(X_{2}\right)}{\partial X_{2}^{1}}, \ldots, \frac{\partial F\left(X_{n}\right)}{\partial X_{n}^{1}}\right], \ldots,\right. \\
& \left.\max \left[\frac{\partial F\left(X_{1}\right)}{\partial X_{1}^{M}}, \frac{\partial F\left(X_{2}\right)}{\partial X_{2}^{M}}, \ldots, \frac{\partial F\left(X_{n}\right)}{\partial X_{n}^{M}}\right]\right\}, \\
\Delta F_{\min }= & \left\{\min \left[\frac{\partial F\left(X_{1}\right)}{\partial X_{1}^{1}}, \frac{\partial F\left(X_{2}\right)}{\partial X_{2}^{1}}, \ldots, \frac{\partial F\left(X_{n}\right)}{\partial X_{n}^{1}}\right], \ldots,\right. \\
& \left.\min \left[\frac{\partial F\left(X_{1}\right)}{\partial X_{1}^{M}}, \frac{\partial F\left(X_{2}\right)}{\partial X_{2}^{M}}, \ldots, \frac{\partial F\left(X_{n}\right)}{\partial X_{n}^{M}}\right]\right\} .
\end{aligned}
$$

For a discrete case, $\Delta F(X)$ is the difference at point $X$ of the fitness function $F(\cdot)$.

By this method, the quantum rotation gate is applied to all probability amplitudes for individual targets in the population. The following equation can be obtained by using the quantum rotation gate $V_{t}$ to update the target $S_{t}$ :

$$
S(t+1)=V(t) \times S(t),
$$

where $V(t)$ is the number $t$ generation of quantum rotation gates. $S(t)$ is the number $t$ probability amplitude for a given object. $S(t+1)$ is the number $(t+1)$ generation probability amplitude of the corresponding object.

Step 5 (perturbation). To solve the problem that CQGA can be prone to be trapped in local optimum, the population requires a perturbation. It is found that through the analysis 


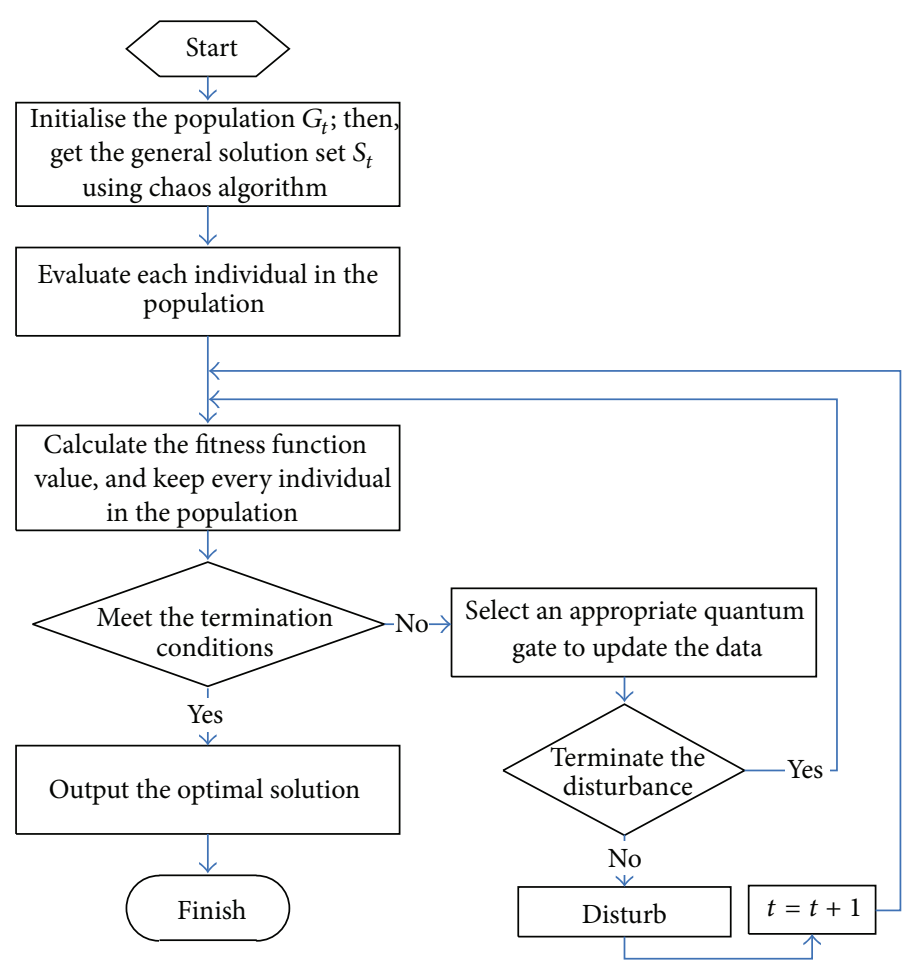

FIGURE 1: Flow chart of the VSS_QGA.

of CQGA, it is very difficult to prevent the best individual in current generation from becoming the local extremum. Therefore, when the best individual does not change in successive generation, the algorithm is trapped into the local extreme. At this point of time, a perturbation must be applied into the population to help it escape from the local optima and start a new search. The complete process of VSS_QGA is shown in Figure 1.

\section{Case Study}

4.1. System Data. In order to verify the effectiveness of the proposed EED and VSS_QGA, a 150-node MG extracted from an actual system in China is used as the test bed, which is shown in Figure 2. The MG system is connected to the main grid via a substation at Bus 0 . The scheduling duration of EED under this study is assumed to be 1 day, which is further divided into 24 time intervals. The technical parameters of different DG technologies in MG are presented in Table 1, while the information of spot prices in each day is given in Table 2.

The prediction of power output for a single WG and $\mathrm{PV}$ is shown in Figure 2. Here, it is assumed that the cutin/rated/cutout speeds of WG units are $v_{\mathrm{CI}}=3 \mathrm{~m} / \mathrm{s}$, $v_{R}=14 \mathrm{~m} / \mathrm{s}$, and $v_{\mathrm{CO}}=25 \mathrm{~m} / \mathrm{s}$, respectively. The variation of wind speed is assumed to follow a Weibull distribution, with the shape parameter $k=2$ and the scale parameter $c=2 \bar{v} / \sqrt{\pi}$, where $\bar{v}$ is the mean value of wind speed in the site. The illumination intensity is simulated based on the Beta distribution, with the parameters $\alpha=0.3$ and $\beta=1.2$. The daily duration of system demand is shown in Figure 3. We take $u_{I}=¥ 0.38 / \mathrm{kW} \cdot \mathrm{h}$, which is consistent with the average feed-in price implemented in China; the emission cost $H_{d e}$ for $\mathrm{SO}_{2}, \mathrm{NO}_{x}$, and $\mathrm{CO}_{2}$ is assumed to be $6.57,8.57$, and $0.02 ¥ / \mathrm{kg}$, respectively. The upper and lower boundaries $p_{\text {gsp. min }}$ and $p_{\text {gsp.min }}$ for the power interacted between DREG and main grid are set to $0 \mathrm{~kW}$ and $200 \mathrm{~kW}$, respectively.

Initial parameter setting has a significant impact on behavior of the algorithm. Therefore, appropriate parameters of VSS_QGA have to be found correctly. According to [14], a large population size could slightly improve the mean cost value, but it will increase the CPU time. Therefore, population size of VSS_QGA is set to 20. Since large angles may cause premature convergence and small angles generally produce better solution, the magnitude of rotation angle $\omega$ is set to $0.02 \pi$. Besides, maximum iteration is $G=800$, qubit length is 20 , and mutation rate is 0.05 .

4.2. Optimization Results. In order to balance the economy and environmental protection in smart MG, $v$ is set to $v=0.5$, which means the contribution rates of both operation and emission cost to the object function are 50\%. VSS_QGA is applied to solve the model of economic and environment dispatch.

Figure 5 gives the optimization results of EED in above system. As can be seen from the power output curves of WT, $\mathrm{PV}$, DICE, and main grid, the wind generation and main grid are the main sources of power supply from 1 a.m. to 7 a.m. After 8 a.m, the energy contribution of PV rises significantly and reaches its peak from 12 a.m. to 3 p.m. At the same time, due to high feed-in price in these periods, DICE units replace the main grid and become the main source to MG. As evening 


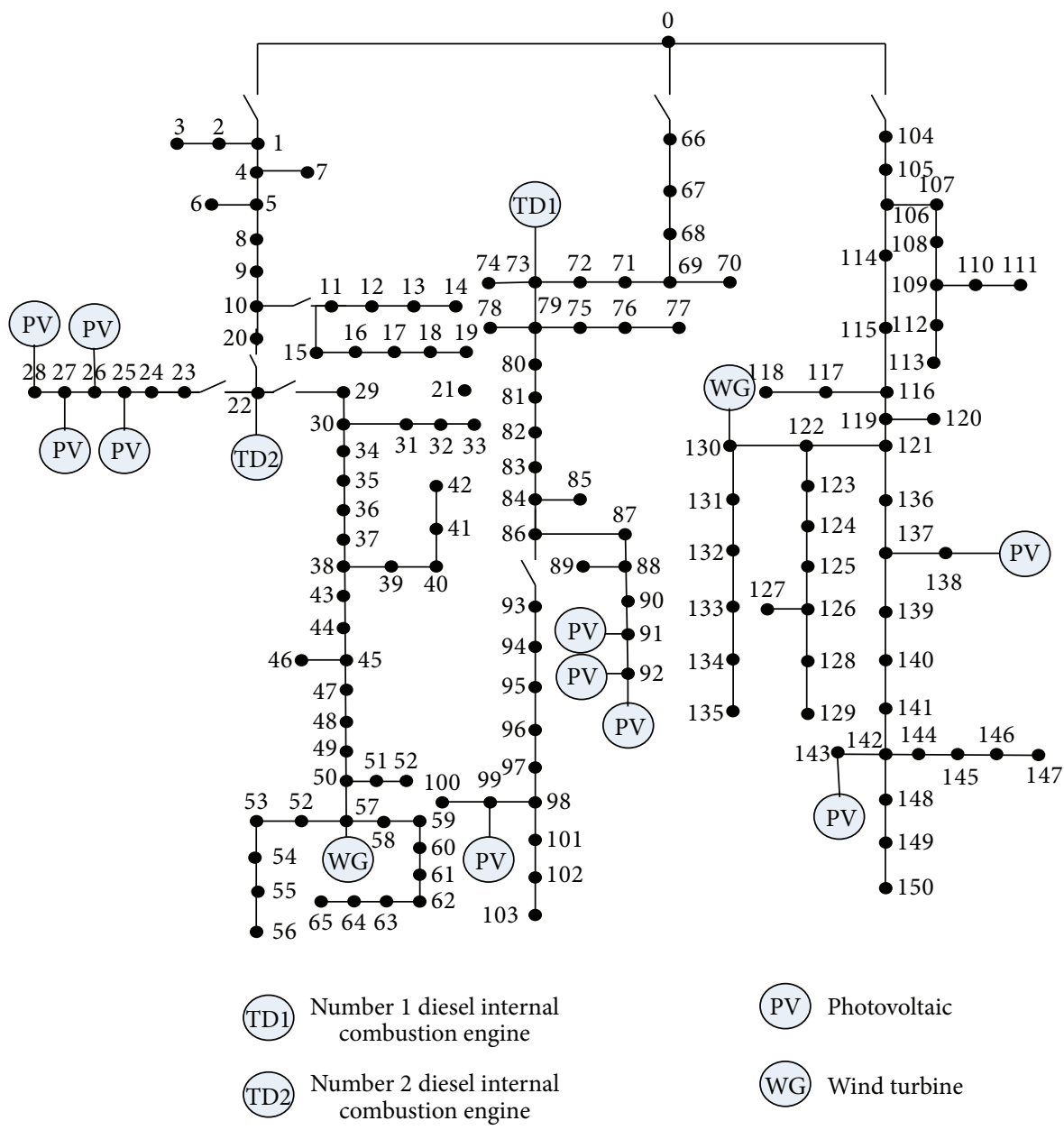

Figure 2: A real 150-node SMG system.

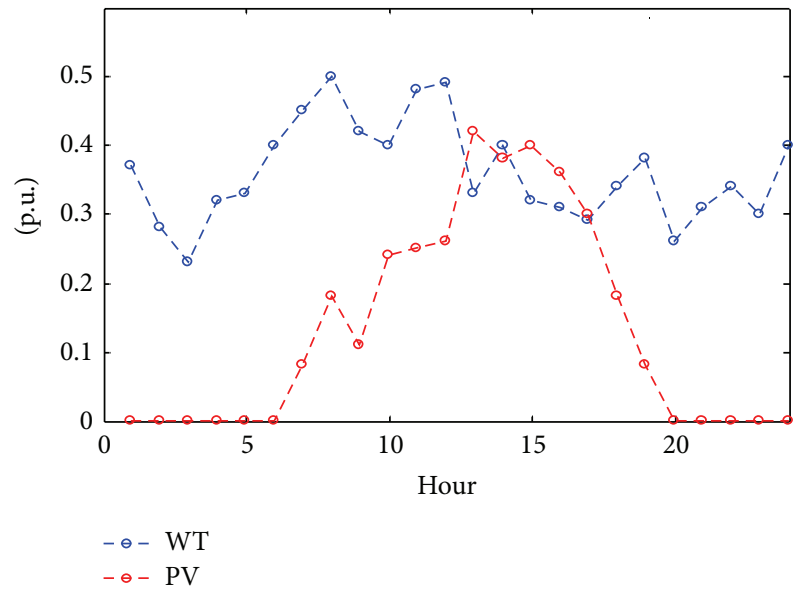

Figure 3: Power forecast of renewable energy generation.

(5 p.m. -7 p.m.) approaches, the energy mix changes again. Wind generation and main grid play dominate roles as wind is strong and the solar output is weakened due to sunset. Last, there is an obvious increase of power generation from DICE

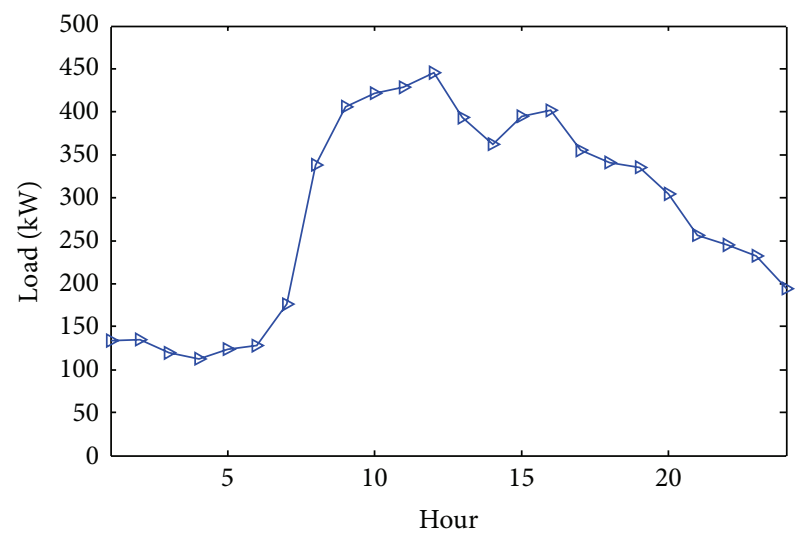

FIgURE 4: Power forecast of load demand.

between 7 p.m. to 9 p.m., implying that these units are put into operation due to the high spot prices in these periods.

When $v$ is 0 , the model in this paper evolves into traditional economic dispatch model with an objective to minimize the expectation operation cost. While $v$ takes a greater value, the model evolves into environmental dispatch 
TABLE 1: Technical data of distributed generation units in SMG.

\begin{tabular}{lcccc}
\hline \multirow{2}{*}{ Type } & \multicolumn{2}{c}{ Operational limits/kW } & Climbing Speeds/(kW·min $\left.{ }^{-1}\right)$ & Number \\
& Lower limit & Upper limit & - & 2 \\
WT & 0 & 100 & - & 10 \\
PV & 0 & 20 & 2 & 1 \\
\#1 DICE & 40 & 100 & 1 & 1 \\
\#2 DICE & 80 & 200 & & 1 \\
\hline
\end{tabular}

TABLE 2: Information of daily spot prices.

\begin{tabular}{lc}
\hline Time/h & Spot price/(¥/(kW·h $))$ \\
\hline 1 & 0.24 \\
2 & 0.18 \\
3 & 0.13 \\
4 & 0.10 \\
5 & 0.13 \\
6 & 0.17 \\
7 & 0.27 \\
8 & 0.39 \\
9 & 0.52 \\
10 & 0.53 \\
11 & 0.81 \\
12 & 1.00 \\
13 & 0.99 \\
14 & 1.49 \\
15 & 0.99 \\
16 & 0.79 \\
17 & 0.40 \\
18 & 0.36 \\
19 & 0.36 \\
20 & 0.41 \\
21 & 0.44 \\
22 & 0.35 \\
23 & 0.30 \\
\hline
\end{tabular}

TABLE 3: Emission characteristics of both traditional power plant and distributed generation $(\mathrm{g} / \mathrm{kW} \cdot \mathrm{h})$.

\begin{tabular}{|c|c|c|c|c|}
\hline & n Type & $\mathrm{SO}_{2}$ & $\mathrm{NO}_{x}$ & $\mathrm{CO}_{2}$ \\
\hline \multirow{4}{*}{ DG } & grid & 0.330 & 2.880 & 643.890 \\
\hline & WT & 0 & 0 & 0 \\
\hline & PV & 0 & 0 & 0 \\
\hline & DICE & 0.464 & 4.331 & 232.037 \\
\hline
\end{tabular}

section with an objective to minimize the emission Table 3. In order to verify that the algorithm in this paper is applicable for all kinds of scheduling problems, though defining $v=0$ and $v=1$, economic dispatch and environmental dispatch model are built and optimized, respectively. Optimization results under different MG scheduling paradigms are as shown in Figure 6.

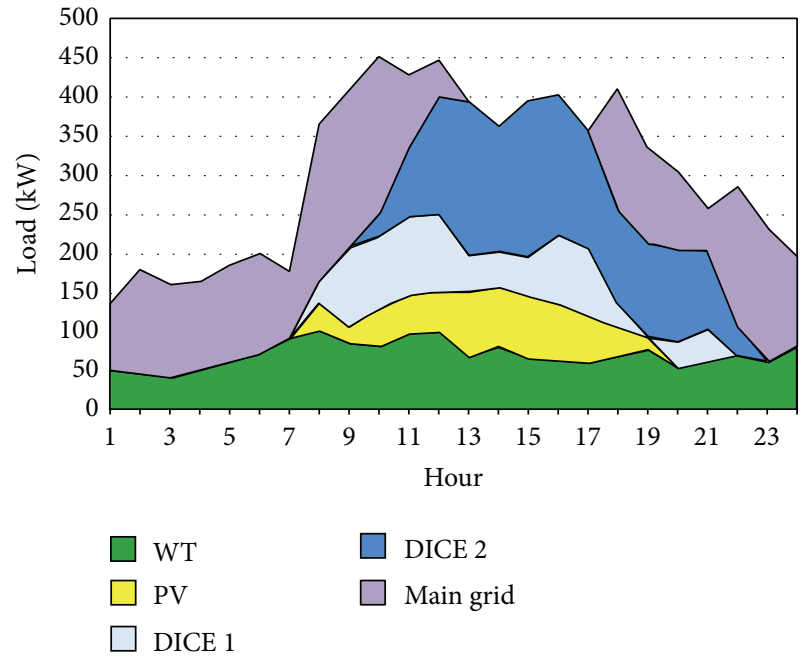

FIGURE 5: Environmental and economic dispatch schemes.

The output of power sources under two different operation modes is shown in Figure 6. As can be seen from Figures 5 and 6 , with the growing of $v$, DERG and main grid output are on the rise, while there is significant fall of power injection from DICE. This is mainly because when $v=0$, that is, only the operating cost is considered, importing energy from the main grid is preferred to the local production because the price of the former is lower in valley periods; thus, DG units offer the extra power that is required. And in the flat and peak periods, the energy contribution of DICE and DREG increases gradually due to their superiority in prices. When $v=1$, that is, only emission cost is considered, the emissionfree DREG units are preferred, which is consistent with our expectation. The optimization results under different MG scheduling paradigms are shown in Table 4.

As is shown in Table 4, the optimal scheme derived from the economic dispatch strategy has the minimum economic cost, which is only $2725.23 ¥$. However, due to lack of consideration on environmental burden in the optimization, the emission cost in such system is dramatically high, which poses a negative effect on the overall attractiveness of the case. When the environmental objective is solely considered, the environmental benefits of MG can be significantly improved. This leads to a drop in the emission cost from DG and the grid by 52.29 and $124.48 ¥$, which corresponds to a decrease of $26.5 \%$ and $73.84 \%$, respectively, compared to the economic dispatch scheme. 
TABLE 4: Optimization results under different MG scheduling paradigms.

\begin{tabular}{|c|c|c|c|c|c|}
\hline Dispatch paradigm & Energy purchase cost & DG generation cost & $\begin{array}{c}\text { Main grid } \\
\text { emission cost }\end{array}$ & DG emission cost & Total cost \\
\hline Economic Dispatch & 782.46 & 1942.77 & 198.72 & 168.59 & 3092.54 \\
\hline Environmental Dispatch & 2259.33 & 1368.22 & 146.43 & 44.11 & 3818.09 \\
\hline $\begin{array}{l}\text { Economic-Environmental } \\
\text { Dispatch }\end{array}$ & 910.46 & 2140.91 & 96.23 & 112.06 & 3259.66 \\
\hline
\end{tabular}

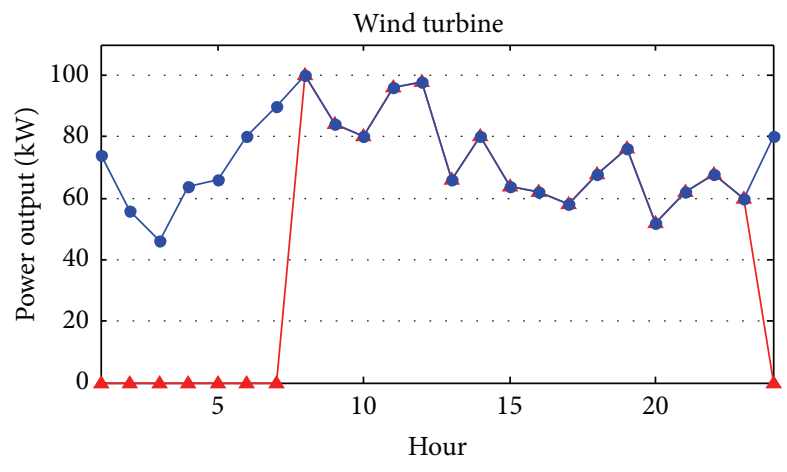

(a)

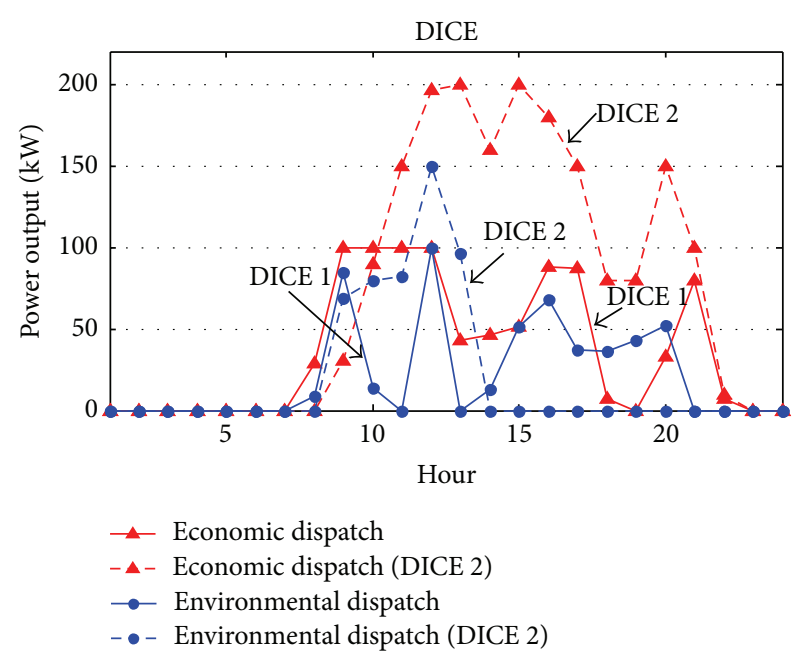

(c)

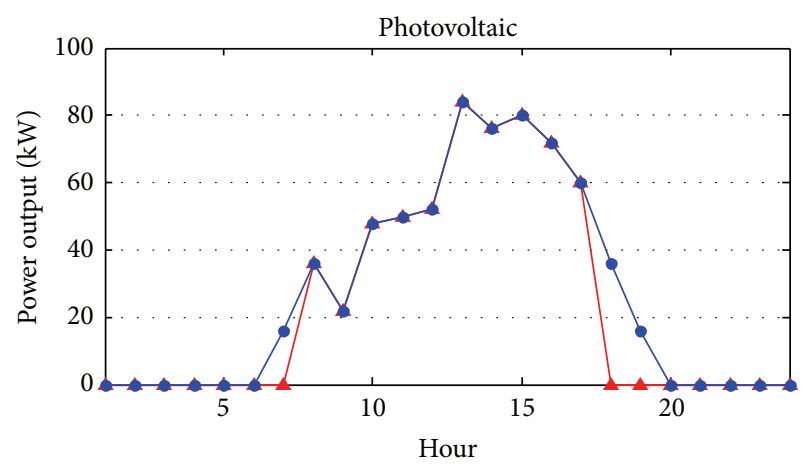

(b)

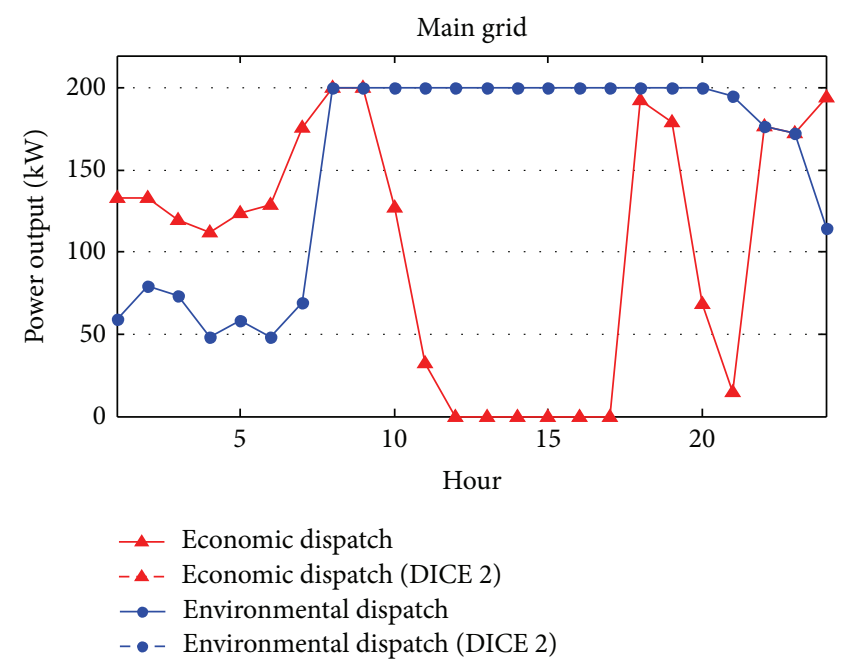

(d)

Figure 6: Power sour output under two different paradigms with $v=0$ and $v=1$.

Under the proposed EED paradigm, the environmental and economic objectives are collectively optimized. The overall cost is calculated to be $3259.66 ¥$, which is the lowest among all scenarios. Although the scheme is not the best in either economic or environmental criteria, the coordination embodied in EED scheme enables superior performance of the plan in terms of comprehensive benefits.

4.3. Discussion. In this section, the convergence and robustness of the proposed optimization method VSS_QGA is evaluated.

To achieve this end, 50 trials are performed with different initial values generated randomly. The objective function value of final schemes in these trails is recorded. Table 5 shows the best, worst, average, and standard deviation (SD), rational standard deviation (\%RSD), and corrected rational standard deviation (\%CRSD) of the results.

As can be seen from Table 5 , there is little difference in the fitness values in the 50 trials and all of them are close to the average. Furthermore, it is also found that \%RSD and \%CRSD are $0.975 \%$ and $0.987 \%$, respectively, which are less than $1 \%$. The above facts demonstrate that the proposed method is not sensitive to initial solution and is able to provide valid dispatch decisions for smart MG close to the global optima in real applications. Through above comparison, the robustness of VSS_QGA is validated.

To evaluate the convergence characteristic of VSS_QGA, different optimization methods are employed to solve EED 
TABLE 5: Summary of different trials in VSS_QGA.

\begin{tabular}{lc}
\hline Parameter & Value \\
\hline Best Cost $(¥)$ & 3204.42 \\
Worst Cost $(¥)$ & 3283.97 \\
Average Cost $(¥)$ & 3259.66 \\
SD & 533.21 \\
\%RSD & 0.975 \\
\%CRSD & 0.987 \\
\hline
\end{tabular}

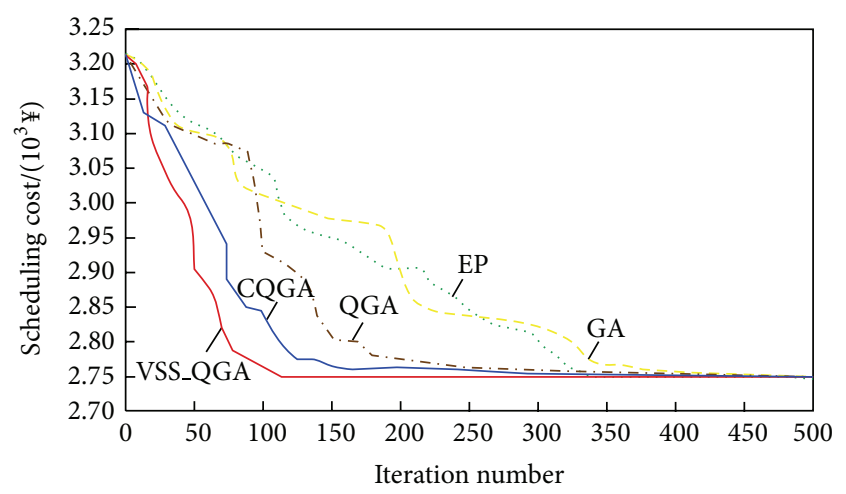

Figure 7: Calculation results of GA, EP, QGA, CQGA, and VSS_QGA.

problem. The comparisons of GA, EP, QGA, CQGA, and VSS_QGA with 500 iterations are given in Figure 7.

As observed, among all above algorithms, VSS_QGA exhibits the best performance. It is obvious that CQGA and VSS_GA have faster convergence speed compared to the rest of methods. This is because unlike the searching strategies of GA, EP, and QGA (which is typically based on the probability to exclude poor solutions by escaping local optimum traps), VSS_QGA and CQGA obtain the optimal solutions by improving chaos motion ergodicity and local search strategy, which is more suitable and efficient for solving the scheduling optimization problem. Different from CQGA, VSS_QGA takes advantage of the variable step size searching and the rotated angle of the fuzzy control strategies, which in turn leads to a superior searching efficiency and convergence performance to reach the global optimal solution.

\section{Conclusions}

In this study, an application of a novel VSS_QGA for EED problem in SMG is developed. The proposed EED model for SMG considers the minimization of generation and emission costs as the objective while taking different constraints such as energy balance, operation limits of power sources, and network characteristics into account. To get faster and better optimization performance, without changing the searching mechanism of the QGA, VSS_QGA introduces the variable step size optimization and the rotation mutational angle fuzzy control method into the algorithm, which improves the evolution speed and enables the optimization immune to the local optima. The effectiveness of the proposed methods is confirmed by comparing the results with the most recently reported literatures, including QGA, EP, and GA. The corresponding results show that the optimal scheduling plan obtained by the proposed EED model will produce greater economic benefit and social benefits, particularly for the large-scale applications. Furthermore, VSS_QGA has superior convergence, robustness, and less computational complexities as compared to other methods.

\section{Conflict of Interests}

The authors declare that there is no conflict of interests regarding the publication of this paper.

\section{Acknowledgment}

This work was supported by the National Key Technology R\&D Program (2013BAA02B02) of China.

\section{References}

[1] C. Chen, S. Duan, J. Yin, and T. Cai, "Energy management system of distributed generation based on power forecasting," Transactions of China Electrotechnical Society, vol. 25, no. 3, pp. 150-156, 2010.

[2] X. Lin, Y. Lu, and L. Wang, "New fault region location scheme in distribution system with DGs," Transactions of China Electrotechnical Society, vol. 23, no. 11, pp. 139-165, 2008.

[3] Y. Xu, L. Zhang, Z. Wang, and X. Li, "Algorithm of service restoration for large area blackout in distribution network with distributed generators," Transactions of China Electrotechnical Society, vol. 25, no. 4, pp. 135-141, 2010.

[4] D. Aydin, S. Özyön, C. Yaşar, and T. Liao, "Artificial bee colony algorithm with dynamic population size to combined economic and emission dispatch problem," International Journal of Electrical Power \& Energy Systems, vol. 54, pp. 144-153, 2014.

[5] C. L. Chen, Z. Y. Chen, and T. Y. Lee, "Multi-area economic generation and reserve dispatch considering large-scale integration of wind power," International Journal of Electrical Power \& Energy Systems, vol. 55, pp. 171-178, 2014.

[6] Q. Niu, H. Zhang, K. Li, and G. W. Irwin, "An efficient harmony search with new pitch adjustment for dynamic economic dispatch," Energy, vol. 65, no. 25, p. 43, 2013.

[7] P. Roy, P. Roy, and A. Chakrabarti, "Modified shuffled frog leaping algorithm with genetic algorithm crossover for solving economic load dispatch problem with valve-point effect," Applied Soft Computing, vol. 13, no. 11, pp. 4244-4252, 2013.

[8] H. T. Jadhav and R. Roy, "Gbest guided artificial bee colony algorithm for environmental/economic dispatch considering wind power," Expert Systems with Applications, vol. 40, no. 16, pp. 6385-6399, 2013.

[9] S. Jiang, Z. Ji, and Y. Shen, "A novel hybrid particle swarm optimization and gravitational search algorithm for solving economic emission load dispatch problems with various practical constraints," International Journal of Electrical Power \& Energy Systems, vol. 55, pp. 629-644, 2014.

[10] Y. C. Liang and J. R. Cuevas Juarez, "A normalization method for solving the combined economic and emission dispatch problem with meta-heuristic algorithms," International Journal of Electrical Power \& Energy Systems, vol. 54, pp. 163-186, 2014. 
[11] S. Jiang, Z. Ji, and Y. Shen, "A novel hybrid particle swarm optimization and gravitational search algorithm for solving economic emission load dispatch problems with various practical constraints," International Journal of Electrical Power \& Energy Systems, vol. 55, pp. 628-644, 2014.

[12] E. Afzalan and M. Joorabian, "Emission, reserve and economic load dispatch problem with non-smooth and non-convex cost functions using epsilon-multi-objective genetic algorithm variable," International Journal of Electrical Power \& Energy Systems, vol. 52, pp. 55-67, 2013.

[13] C. Yaşar and S. Özyön, "Solution to scalarized environmental economic power dispatch problem by using genetic algorithm," International Journal of Electrical Power \& Energy Systems, vol. 38, no. 1, pp. 54-62, 2012.

[14] G.-C. Liao, "Solve environmental economic dispatch of Smart MicroGrid containing distributed generation system-Using chaotic quantum genetic algorithm," International Journal of Electrical Power \& Energy Systems, vol. 43, pp. 779-787, 2012.

[15] A. D. S. Nicolau, R. Schirru, and A. Alvarenga De Moura Meneses, "Quantum evolutionary algorithm applied to transient identification of a nuclear power plant," Progress in Nuclear Energy, vol. 53, no. 1, pp. 86-91, 2011.

[16] G. S. Sailesh Babu, D. Bhagwan Das, and C. Patvardhan, "Realparameter quantum evolutionary algorithm for economic load dispatch," IET Generation, Transmission and Distribution, vol. 2, no. 1, pp. 22-31, 2008.

[17] A. M. Martínez, "Recognizing imprecisely localized, partially occluded, and expression variant faces from a single sample per class," IEEE Transactions on Pattern Analysis and Machine Intelligence, vol. 24, no. 6, pp. 748-763, 2002. 


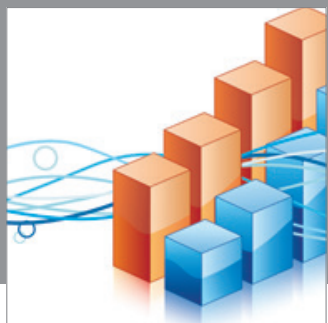

Advances in

Operations Research

mansans

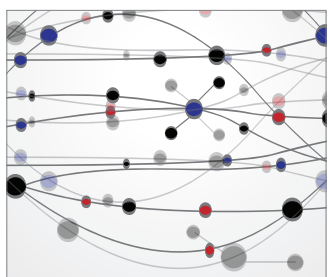

The Scientific World Journal
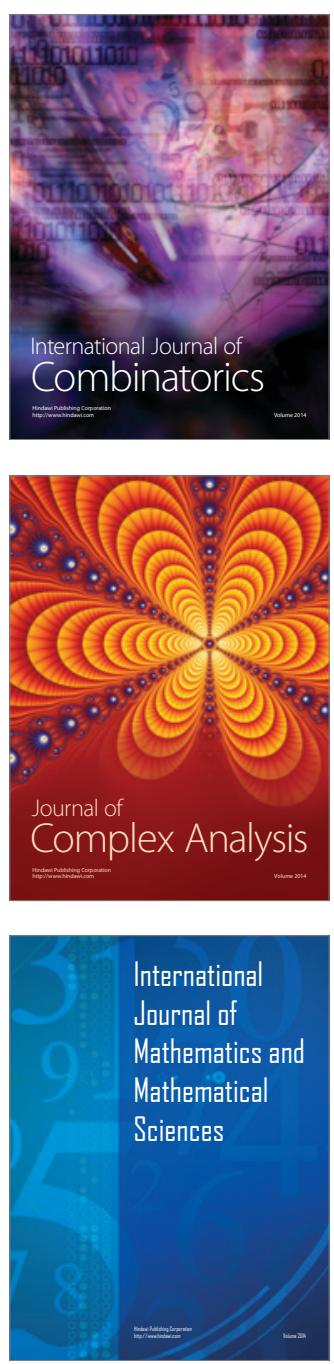
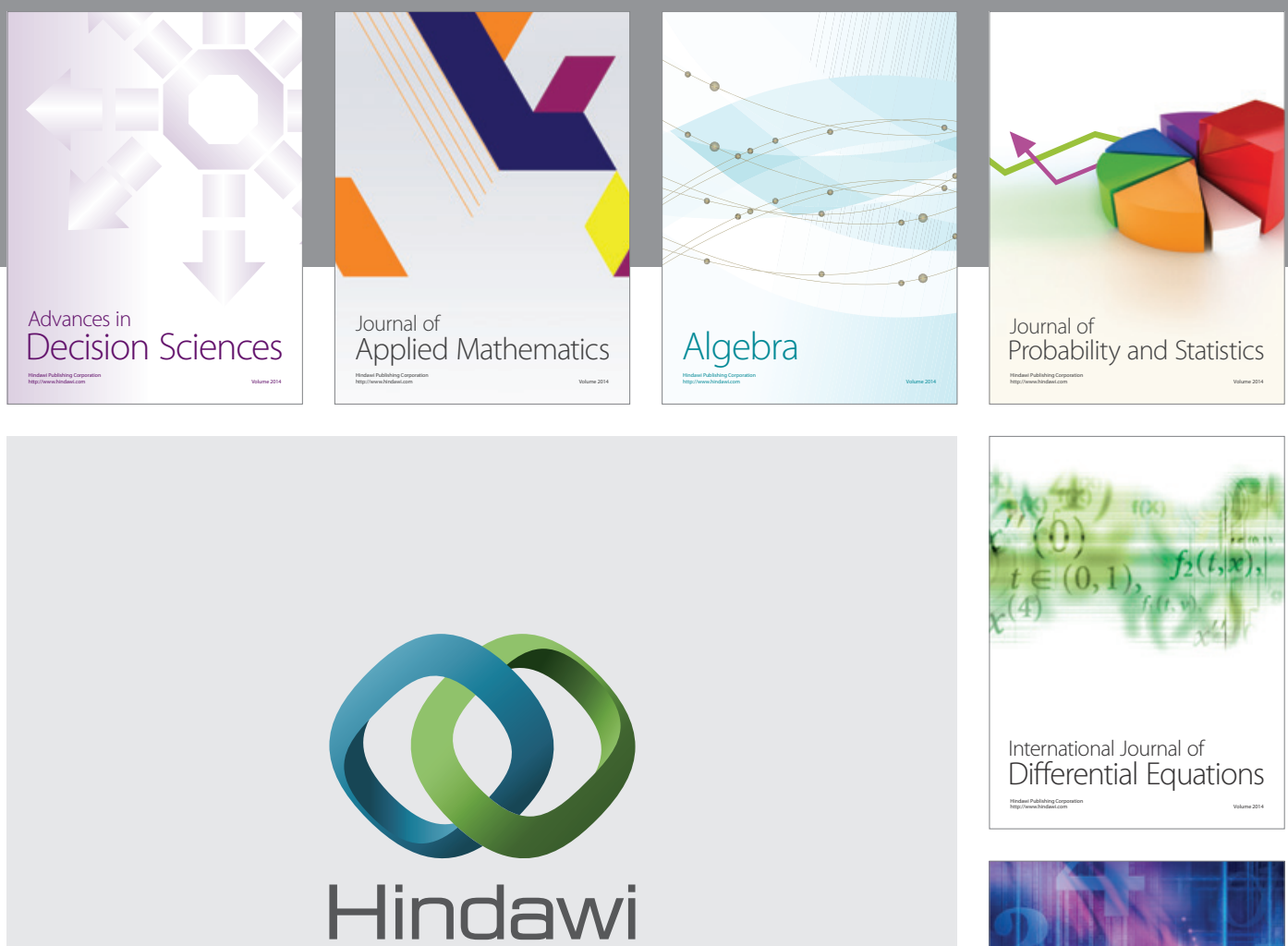

Submit your manuscripts at http://www.hindawi.com
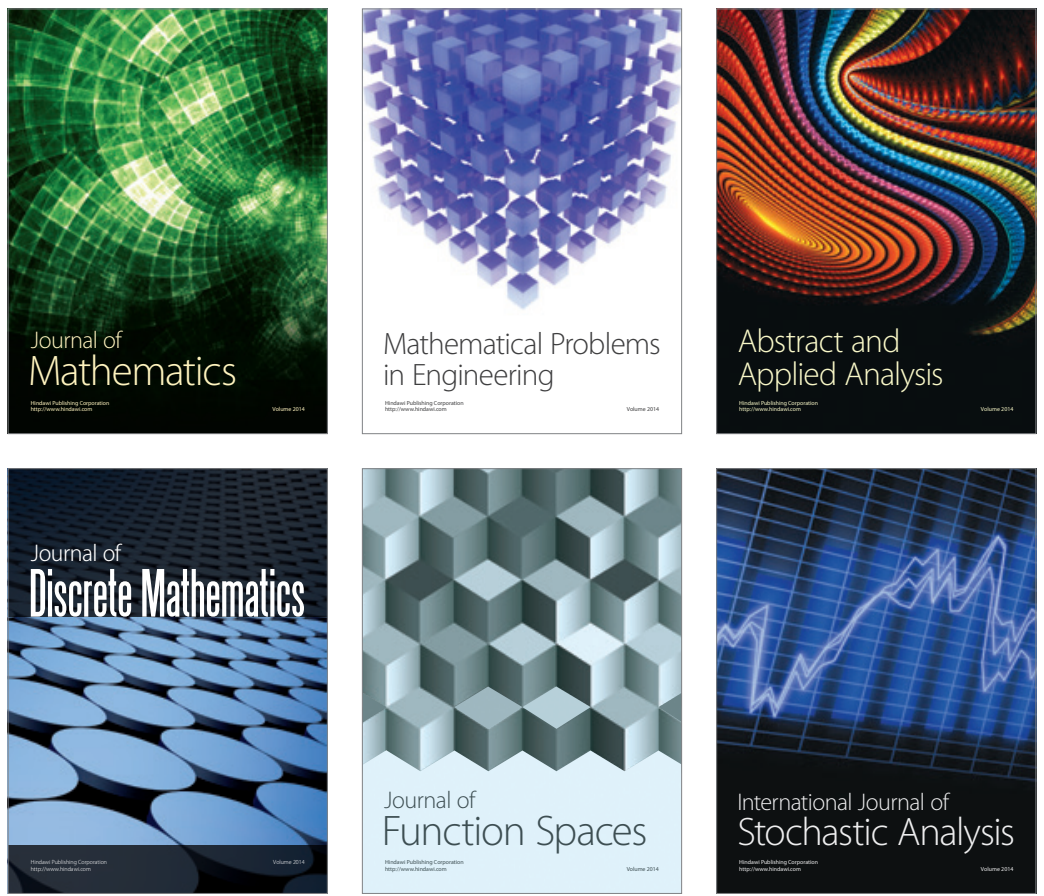

Journal of

Function Spaces

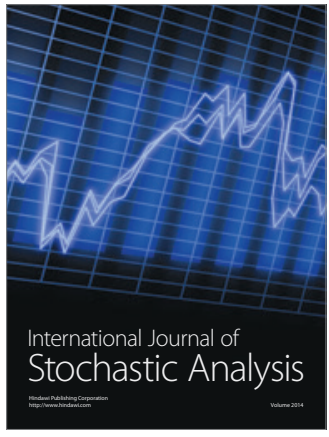

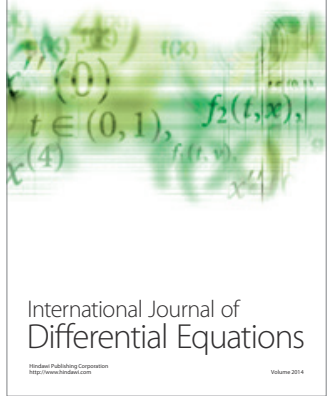
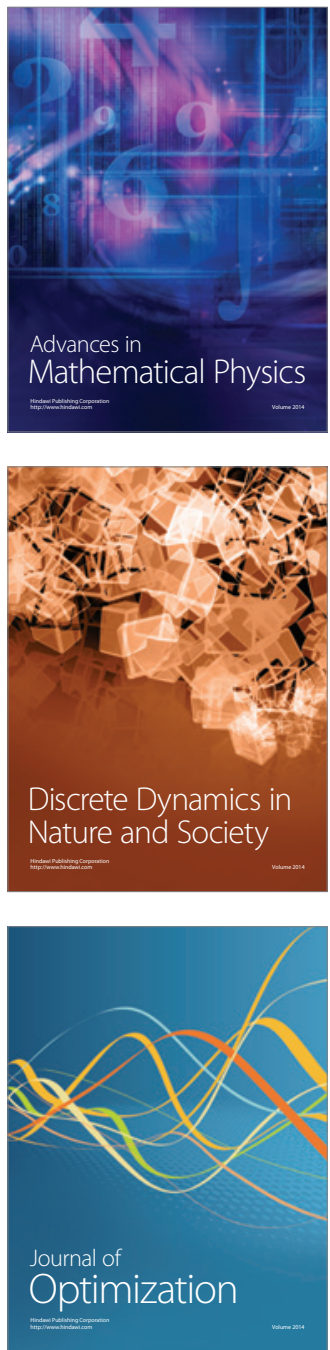